\title{
Dual Cobalt and Photoredox Catalysis Enabled Intermolecular Oxidative Hydrofunctionalization
}

\author{
Han-Li Sun, Fan Yang, Wei-Ting Ye, Jun-Jie Wang and Rong Zhu* \\ Beijing National Laboratory for Molecular Sciences (BNLMS), Key Laboratory of Bioorganic Chemistry and Molecular \\ Engineering of Ministry of Education, College of Chemistry and Molecular Engineering, Peking University, Beijing 100871, \\ China \\ Supporting Information Placeholder
}

\begin{abstract}
A general protocol has been developed for the Markovnikov-selective intermolecular hydrofunctionalization based on visible-light-mediated $\mathrm{Co} / \mathrm{Ru}$ dual catalysis. The key feature involves the photochemical oxidation of an organocobalt(III) intermediate derived from hydrogen atom transfer, which is supported by electrochemical analysis, quenching studies and stoichiometric experiments. This unique redox process enables the efficient branch-selective alkylation of pharmaceutically important nucleophiles (phenols, sulfonamides and various $N$-heterocycles) using a wide range of alkenes including moderately electron-deficient ones. Moreover, light-gated polar functionalization via organocobalt species was demonstrated.
\end{abstract}

The Brønsted acid-mediated intermolecular Markovnikov addition has been known for more than a hundred years. ${ }^{1}$ On paper, it offers a straightforward and modular approach to many pharmaceutically relevant structures such as branched-alkyl-substituted phenols and $N$-heterocycles, without resorting to pre-activated coupling partners or subsequent multi-step conversions (Scheme 1a). ${ }^{2}$ Not surprisingly, such transformations in practice are often complicated by the thermodynamically disfavored protonation process and high-energy carbocations involved. ${ }^{3}$ To this, chemists have been searching for catalytic alternatives mimicking this process in a more controlled fashion. ${ }^{4}$ Guided by the rich literatures on the first-row transition metal, ${ }^{5}$ in particular the cobalt-catalyzed hydroelementations via hydrogen atom transfer (HAT), ${ }^{6}$ we recently introduced an I(III)-promoted intermolecular oxidative hydrofunctionalization reaction catalyzed by a cobalt salen complex. ${ }^{7}$ Highly chemo- and regio-selective additions to unactivated terminal olefins and electron-rich styrenes have been realized with a number of heteroatom-based nucleophiles, which includes carboxylic acids, phenols, and sulfonamides. More recently, benzotriazoles have also been added as viable reaction partners by Yahata and Akai. ${ }^{8}$ A key mechanistic hypothesis of this transformation involves the oxidation of an organocobalt(III) intermediate $\mathbf{A}$ into a $\mathbf{C o}(\mathrm{IV})$ species $\mathbf{B}$, an organometallic radical cation that displays polar reactivity (Scheme 1b). ${ }^{9}$ Our studies suggested that a $\mathrm{Co}(\mathrm{III})-\mathrm{X}$ complex $(\mathrm{X}=$ counterion/anionic ligand) $\mathbf{C}$ likely functions as the oxidant for this process, whose reduction potential lies close to the oxidation potential of $\mathbf{A}$.

As part of the continuing effort exploring the oxidative functionalization of organocobalt complexes in catalysis by a mechanistic-driven approach, it came to our attention that such reversible electron transfer between cobalt species could be a major limiting factor. When either the oxidation of $\mathbf{A}$ or the following nucleophilic trapping of $\mathbf{B}$ is sluggish, significant increase in the concentration of $\mathbf{A}$ is anticipated, which would eventually lead to traditional radical-based reactions via $\mathrm{Co}-\mathrm{C}$ bond homolysis. For instance, the resulting radical $\mathbf{D}$ once escaped from a solvent cage could dimerize, abstract a halogen atom, or react with trace dioxygen. It is thus predicted that the reaction efficiency would be largely limited by the electronic property of the alkyl group in $\mathbf{A}$ and the nucleophilicity of the reaction partner. ${ }^{10}$ In other words, the outcome would be highly substrate-dependent. Indeed, we observed contrasting behaviors of $p$-methoxystyrene (2a) and p-chlorostyrene (2b) under the standard reaction conditions (Scheme 1c). The former produced Markovnikov adduct in quantitative yield, while the latter virtually only underwent reductive dimerization.

In order to overcome this limitation by a general catalyst-controlled solution, a natural thought would be to modify the structure of the cobalt salen complex. However, such approach would likely lead to marginal effect because both $\mathbf{A}$ and $\mathbf{C}$ are subjected to change in a rather similar way. Therefore, we sought an alternative mechanism involving a stronger catalytic oxidizing species that is compatible with the HAT conditions. In this work, we report the realization of this approach via a merger of a photoredox cycle with HAT catalysis. The dual catalysis significantly expands the viable redox window, and thus leads to an efficient and general single-step entry into a range of pharmaceutically relevant structures. The regioselectivity and scope of this protocol complement established methodologies, for example the anti-Markovnikov dehydrogenative hydrofunctionalizations via cobaloxime catalysis. ${ }^{11}$ In addition, we provide a rare example where the polar reactivity of an organocobalt intermediate was gated by external physical stimuli.

To assist the design, we began by evaluating the electrochemical properties of cobalt salen complexes $\mathbf{1 a}$ and $\mathbf{1 b}$, as well as an alkylcobalt(III) complex $\mathbf{6}$ bearing an isopropyl group as a model for $\mathbf{A}$ (Scheme 1c). ${ }^{12}$ The $E_{1 / 2}$ for the $1 \mathbf{a}^{+} / \mathbf{1 a}$ redox couple locates at $-0.02 \mathrm{~V}$ vs $\mathrm{Fc}^{+} / \mathrm{Fc}$ in dichloromethane, and is slightly lower than that of the $1 \mathbf{b}^{+} / \mathbf{1 b}$ couple $(0.11 \mathrm{~V}) .{ }^{13}$ Complex $\mathbf{6}$ displayed an irreversible oxidation wave at $\mathrm{E}_{\mathrm{p} / 2}=-0.01 \mathrm{~V}$ vs $\mathrm{Fc}^{+} / \mathrm{Fc}$, likely corresponding to the $\mathrm{R}-\mathrm{Co}(\mathrm{III}) / \mathrm{R}-\mathrm{Co}(\mathrm{IV})$ oxidation based on literature reports. $.^{9 a}, 14$ The measured potentials corroborates the previous mechanistic hypothesis regarding the SET between Co complexes, which was further supported by a titration experiment. ${ }^{15}$ It is noted that the Co complexes undergo additional redox events at both more positive and negative parts of the spectrum. Ideally, a photoredox catalyst should not trigger such undesired processes.

Guided by the electrochemical data, we envisioned that the excited state of $\mathrm{Ru}(\mathrm{bpy}) 3^{2+}$ (bpy $=2,2$ '-bipyridyl, $\mathrm{E}_{1 / 2}\left[\mathrm{Ru}^{\mathrm{II}} / \mathrm{Ru}^{\mathrm{I}}\right]=0.77 \mathrm{~V}$ vs SCE in acetonitrile) might allow for the efficient oxidation of organocobalt(III) complex A. ${ }^{16}$ A proposed catalytic cycle is depicted in Scheme 1e. It commences with the formation of $\mathbf{A}$ via HAT between a $\mathrm{Co}-\mathrm{H}$ species and an alkene 2 followed by cage collapse. The key step thus involves the facile reductive quenching of visible light-generated $* \mathrm{Ru}$ (II) by $\mathbf{A}$, which would efficiently produce the electrophilic Co(IV) complex $\mathbf{B}$ with minimal reverse reaction. $\mathbf{B}$ is subsequently trapped by a nucleophile to give the Markovnikov adduct. The resulting $\mathrm{Ru}(\mathrm{I})$ and $\mathrm{Co}(\mathrm{II})$ complexes then react with a stoichiometric two-electron oxidant, either the hypervalent iodine reagent $\mathbf{3}$ or an $N$-fluoropyridinium salt $\mathbf{7}$, to 
Scheme 1. Mechanistically-Guided Design of a Dual-Catalysis Approach to Broad-Spectrum Oxidative Hydrofunctioalization.

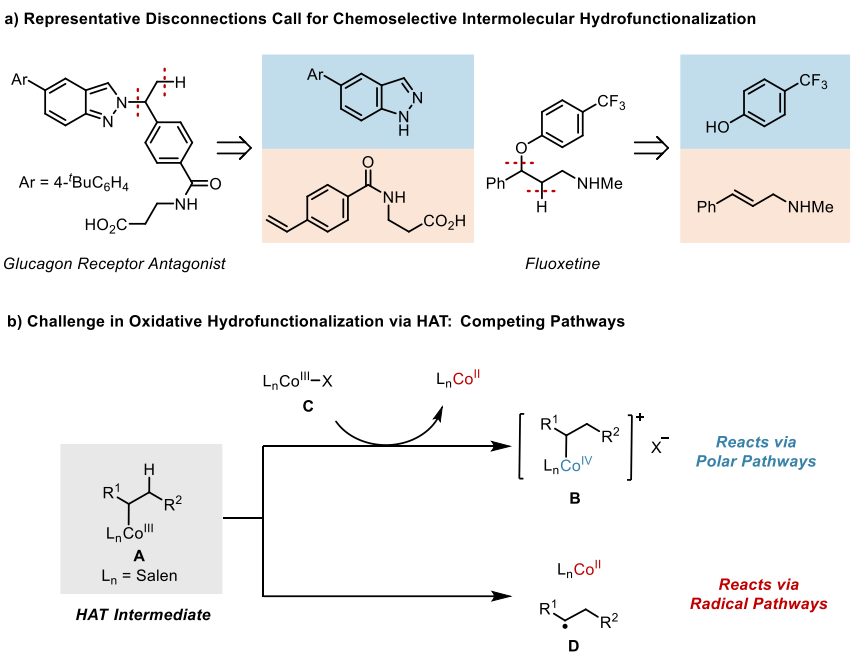

c) Example: Major Limitations Posed by Mismatched Redox Properties

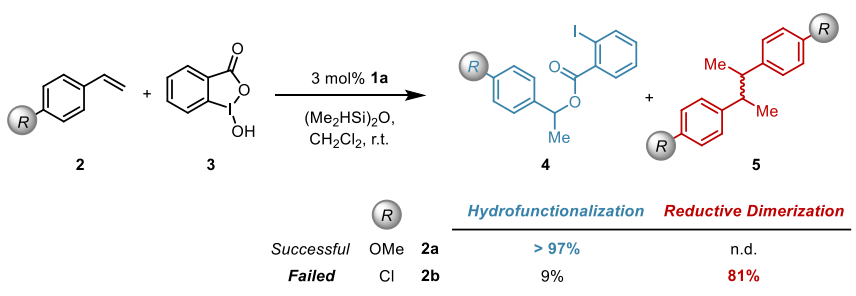

regenerate $\mathrm{Ru}(\mathrm{II})$ and $\mathrm{Co}(\mathrm{III})$ species, respectively. The latter finally reacts with a silane and return to the inital $\mathrm{Co}-\mathrm{H}$ complex.

To test this hypothesis, we first evaluated the oxidative HAT hydrofunctionalization reaction of $\mathbf{2} \mathbf{b}$ with $\mathbf{3}$ and a silane at room temperature (Table 1). Compared to the original reaction catalyzed solely by $3 \mathrm{~mol} \%$ Co complex $1 \mathrm{a}$ (entry 1 ), the addition of $1 \mathrm{~mol} \%$ $\mathrm{Ru}$ (bpy) ${ }_{3} \mathrm{Cl}_{2} \bullet 6 \mathrm{H}_{2} \mathrm{O}$ accompanied by blue LED irradiation effectively suppressed the reductive dimerization and inverted the selectivity completely, affording $\mathbf{4 b}$ in over $80 \%$ yield (entry 2 ). The dark reaction using $1 \mathbf{b}$ gaves $30 \%$ yield of $\mathbf{4 b}$, presumably due to the slightly higher $\mathrm{E}_{1 / 2}$ of $\mathbf{1} \mathbf{b}^{+} / \mathbf{1} \mathbf{b}$ (entry 3 ). The combination of $1 \mathrm{~b}$ and the photoredox conditions further pushed the reaction to quantitative conversion without observing any dimer formation (entry 4). Control experiments under light irradiation leaving out the Co or Ru catalyst confirmed the essential roles of both (entries $5-7)$.

We next examined a few alternative metal-based photoredox catalysts at wavelengths close to their individual absorption maximums, respectively. For example, $\mathrm{Ru}(\mathrm{bpz}) 3^{2+}(\mathrm{bpz}=$ 2,2'-bipyrazine), which possesses a highly oxidizing excited state $\left(\mathrm{E}_{1 / 2}\left[* \mathrm{Ru}^{\mathrm{II}} / \mathrm{Ru}\right]=1.45 \mathrm{~V}\right.$ vs SCE$)$, was found incompatible (entry 8). On the other end of the redox spectrum, $f a c-\operatorname{Ir}(\mathrm{ppy})_{3}$ (ppy $=$ 2-phenylpyridine) did not promote the desired transformation either (entry 9). This could be attributable to either the strongly reducing nature $\left(\mathrm{E}_{1 / 2}\left[\mathrm{Ir}^{\mathrm{IV}} / * \mathrm{Ir}^{\mathrm{II}}\right]=-1.73 \mathrm{~V}\right.$ vs $\left.\mathrm{SCE}\right)$ or the high triplet state energy $(57.8 \mathrm{kcal} / \mathrm{mol})$ that interferes with the HAT cycle, which also indicates that energy transfer catalysis is unlikely involved in the productive mechanism. ${ }^{17}$ Therefore, $\mathrm{Ru}(\mathrm{bpy}) 3^{2+}$ seems to hit a sweet spot with regard to redox matching, which is consistent with the observation made with a different Ru catalyst of similar redox properties (entry 10). Finally, the loading of $\mathbf{1 b}$ in the dual catalyst system could be reduced to $1 \mathrm{~mol} \%$ without compromising the yield (entry 11).

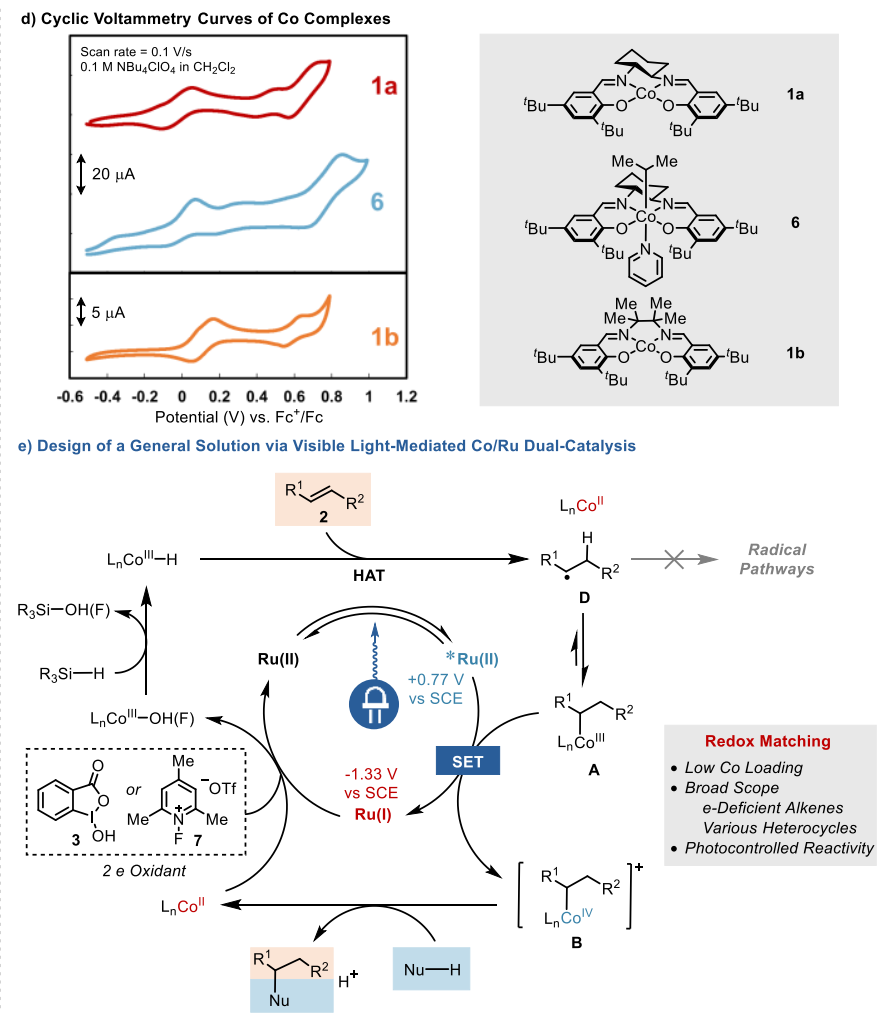

Table 1. Evaluation of the effect of photoredox catalysts. ${ }^{a}$

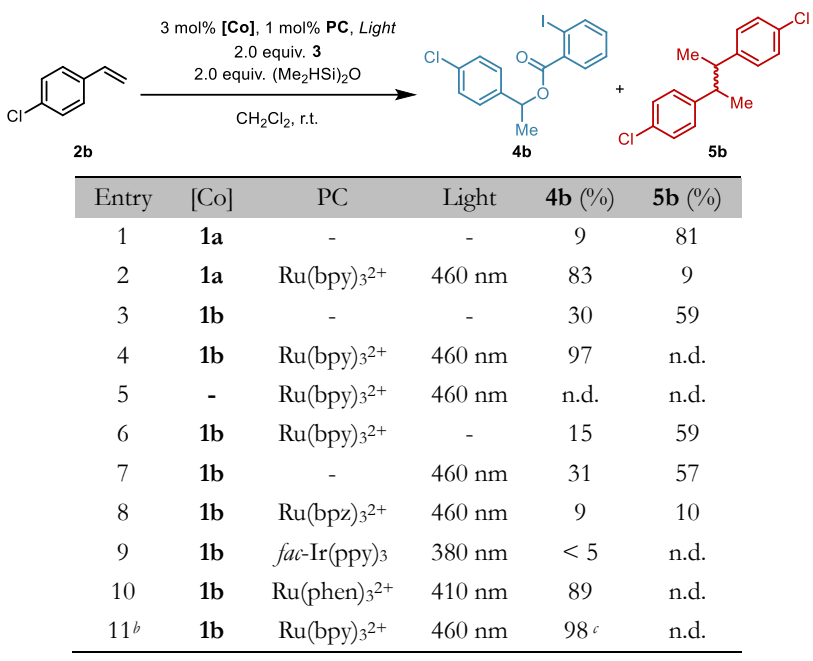

aStandard conditions: $2 \mathbf{b}(0.10 \mathrm{mmol}), \mathbf{1 b}(3 \mathrm{~mol} \%)$, photocatalyst $(1 \mathrm{~mol} \%)$, $\left(\mathrm{Me}_{2} \mathrm{SiH}\right)_{2} \mathrm{O}(0.20 \mathrm{mmol}), 3(0.20 \mathrm{mmol})$ in $1.0 \mathrm{~mL} \mathrm{CH}_{2} \mathrm{Cl}_{2}$ at r.t. for $18 \mathrm{~h}$. The yields were determined by ${ }^{1} \mathrm{H}$ NMR analysis of the crude reaction mixture. The yields of $\mathbf{5} \mathbf{b}$ refer to the corresponding $\mathbf{2} \mathbf{b}$ converted. See Table S2 for details. ${ }^{b}$ 2a $(0.30 \mathrm{mmol}), \mathbf{b}(1 \mathrm{~mol} \%)$, photocatalyst $(1 \mathrm{~mol} \%),\left(\mathrm{Me}_{2} \mathrm{SiH}\right)_{2} \mathrm{O}(0.60 \mathrm{mmol})$, $3(0.60 \mathrm{mmol})$ in $1.0 \mathrm{~mL} \mathrm{CH}_{2} \mathrm{Cl}_{2}$ at $25{ }^{\circ} \mathrm{C}$ for $36 \mathrm{~h}$. Isolated yield.

The divergent reactivities observed in the presence/absence of light prompted us to compare their kinetic profiles (Figure 1a). The light-mediated reaction displayed substantially greater rate throughout the course of the reaction (plot 1). In contrast, induction periods of different lengths were observed for both the formation of $\mathbf{4 b}$ (plot 3 ) and $\mathbf{5 b}$ (plot 4 ) in the absence of light. Interestingly, the reductive dimerization started to take off at $\mathrm{t}=4$ $\mathrm{h}$, then rapidly overwhelmed the desired transformation, which seems to reflect a dynamic equilibrium involving cobalt species. The light-controlled switch was clearly illustrated by a reaction 
carried out with intermitent irradiation (plot 2, light exposure between $t=4-6,7.5-9 \mathrm{~h}$ ). It is well-known that the homolysis of an organocobalt(III) can be photochemically controlled, a feature that is particularly useful for radical polymerizations. ${ }^{18}$ However, light-regulation of polar reactivity has been essentially unexplored. ${ }^{19}$ To this, our dual catalyst system seems to provide an interesting demonstration.

To further probe whether such dramatic improvement stems from the photochemical oxidation of the organocobalt(III) intermediate A as proposed, Stern-Volmer quenching studies were carried out (Figure $1 \mathrm{~b}$ ). The excited state $* \mathrm{Ru}$ (II) was found to be readily quenched by the model compound $\mathbf{6}$, but not the stoichiometric oxidant 3. This is consistent with the hypothesized reductive quenching process. In addition, mixing $\mathbf{6}$ and $\mathbf{3}$ either in the presence or absence of the Ru catalyst in dark resulted in no oxidative functionalization product $\mathbf{8}$ as expected from measured electrochemical data (Figure 1c). However, when the photocatalyst-containing mixture was exposed to blue light, 8 was formed in $15 \%$ yield. Collectively, these results strongly support that an analogous oxidation of $\mathbf{A}$ by $* \mathrm{Ru}(\mathrm{II})$ might take place in the catalytic reaction.
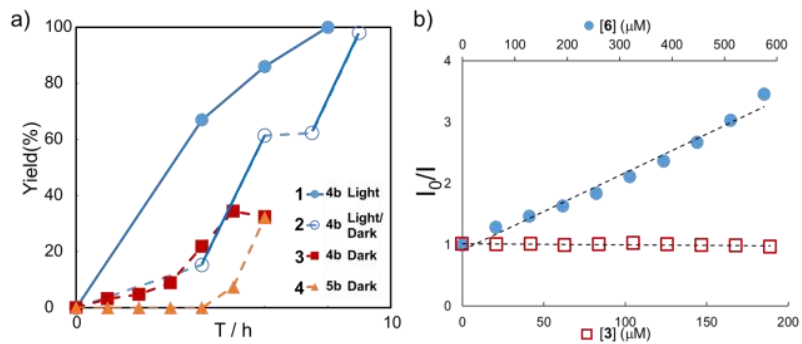

c)

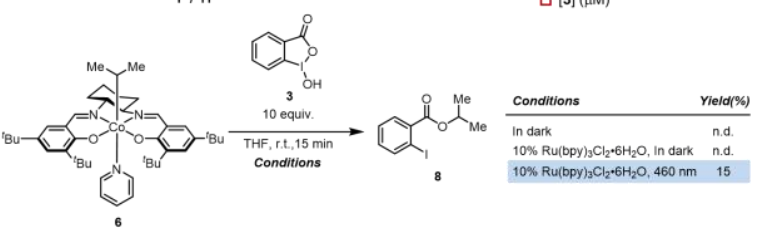

Figure 1. Mechanistic Studies. a) Reaction profiles. Conditions: $\mathbf{2 b}$ (1.0 equiv.), 3 (2.0 equiv.), $\left(\mathrm{Me}_{2} \mathrm{SiH}\right)_{2} \mathrm{O}$ (2.0 equiv.) at r.t. in $\mathrm{CH}_{2} \mathrm{Cl}_{2} \bullet \bullet / 0: 3 \% 1 \mathbf{b}, 1 \%[\mathrm{Ru}$; -/ $\mathbf{\Lambda}: 3 \% \mathbf{1 b}$ only. Solid lines: exposed to $460 \mathrm{~nm}$ light; dash lines: in the absence of light. b) Stern-Volmer quenching studies of Ru(bpy) $3^{2+}$ in the prescence of $\mathbf{3}$ or $\mathbf{6}$. c) Stoichiometric experiments showing that photochemical oxidation of 6 led to oxidative functionalization product. The yields were determined by ${ }^{1} \mathrm{H}$ NMR analysis of the crude reaction mixtures.

Scheme 2. Scope of the Dual-Catalyzed Oxidative Hydrofunctionalization via HAT. ${ }^{a}$

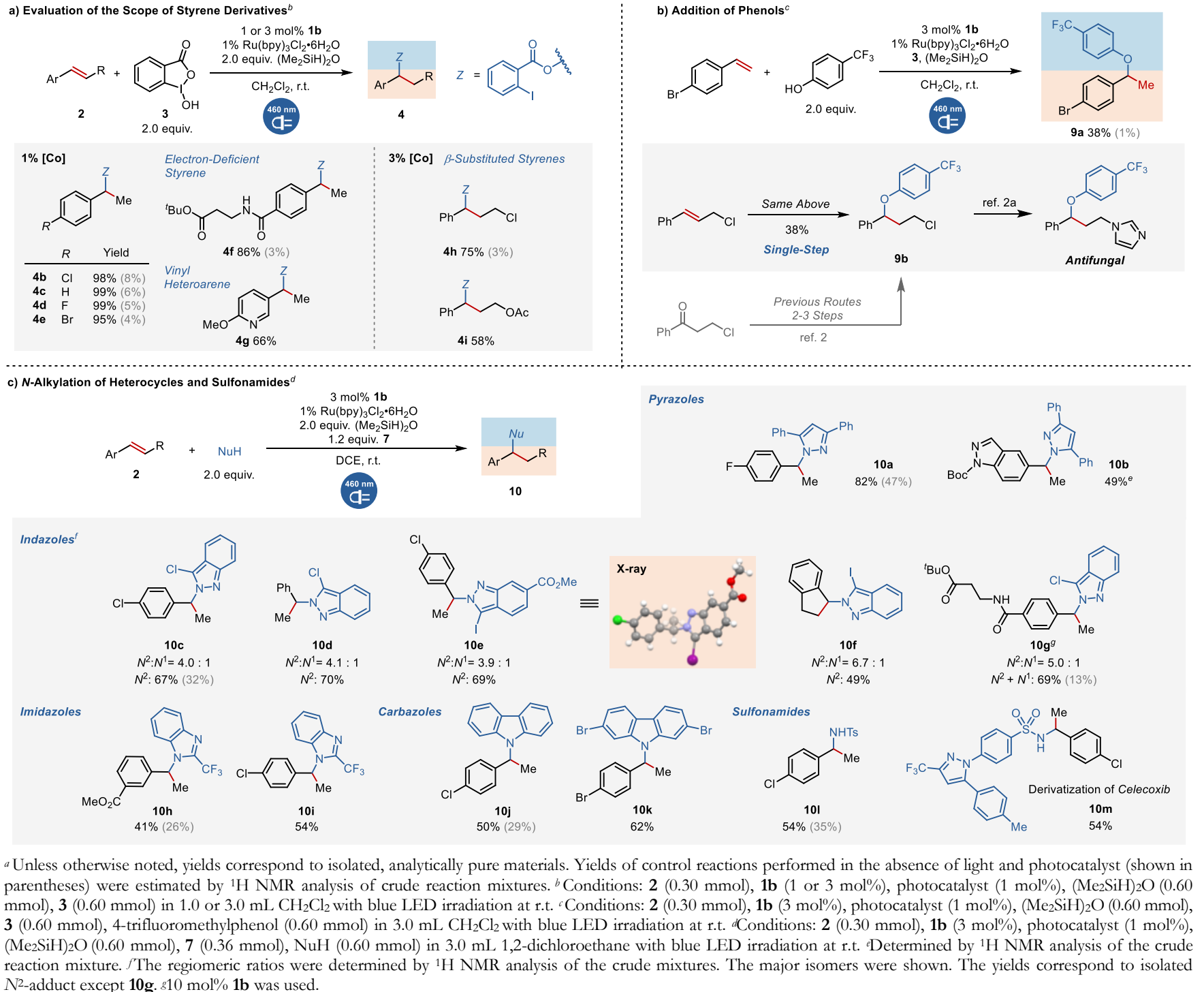


With these results in hand, we set out to apply the new dual catalysis protocol to a series of vinylarene substrates (Scheme 2a). They typically failed or led to very low turnover number under previous oxidative hydrofunctionalization conditions as indicated by the results of control experiments shown in parentheses. Quantitative to good yields were obtained for styrenes, $\beta$-substituted styrenes and a vinylpyridine derivative (4b-i). In all cases, no reductive dimerization was detected. It is worth noting that a moderately electron-withdrawing amide group at the para-position is well tolerated, showcasing the significantly expanded redox window brought by the photoredox cycle $(\mathbf{4 f}) .{ }^{20}$ The fact that such alkenes can be functionalized directly represents an additional notable advantage over traditional acid-mediated processes besides chemoselectivity.

This protocol proved readily amenable for phenol addition (Scheme 2b). The yields were moderate because of the competing addition of 2-iodobenzoic acid. Nonetheless, it allows for single-step modular assembly of useful structures that would otherwise require multi-step synthesis. For instance, starting from simple cinnamyl chloride, one could rapidly access $\mathbf{9 b}$, which is a common intermediate en route to a series of biologically active molecules.2a, b While the highly reactive allylic $\mathrm{C}-\mathrm{Cl}$ bond in cinnamyl chloride is typically susceptible to nucleophiles including low-valent transition metal complexes, it did not interfere with the nucleophile addition under the oxidative hydrofunctionalization conditions.

To further demonstrate the synthetic utility and generality of this method, we next show that a reasonably broad scope of nitrogen-based heterocycles can be efficiently alkylated using 3 $\mathrm{mol} \%$ catalyst $\mathbf{1 b}$ (Scheme $2 \mathrm{~b}$ ). This includes pyrazoles (10a-b), indazoles $(\mathbf{1 0} \mathbf{c}-\mathbf{g})$, imidazoles $(\mathbf{1 0 h}-\mathbf{i})$ and carbazoles $(\mathbf{1 0 j}-\mathbf{k})$. To avoid a competing nucleophile, we replaced 3 by $\mathrm{N}$-fluorotrimethylpyridinium triflate (7) as the stoichiometric oxidant, ${ }^{21}$ which does not change the rest of the catalytic cycles and indeed led to similar trends. These heterocycles were challenging nucleophiles under previous conditions even in the cases involving electron-rich/neutral styrenes (yields in parentheses). Dual catalysis offered doubled yields on average. It is noted that the thermodynamically less favored $N^{2}$-alkylation was the major pathway for indazoles. ${ }^{22}$ It complements existing $\mathrm{S}_{\mathrm{N}} 2$ chemistry and other transition metal-catalyzed methods in regioselectivity, which could potentially find use in facilitating drug-discovery processes. ${ }^{23}$ Improved turnovers were also observed for sulfonamide $N$-alkylations, which enabled the late-stage modification of a drug molecule $(\mathbf{1 0 1}-\mathbf{m})$. Finally, the excellent chemoselectivity of the process is demonstrated by a range of functional groups present besides the heterocycles themselves, such as activated aryl iodides (10e) and acid-sensitive protecting groups $(\mathbf{1 0 b}, \mathbf{g})$.

In summary, we have developed an efficient and general protocol for the Markovnikov-selective intermolecular hydrofunctionalization of styrenes and vinylheteroarenes including moderately electron-deficient ones. The key design is the visible light-mediated $\mathrm{Co} / \mathrm{Ru}$ dual catalysis that stems from electrochemical analysis. It enables the previously difficult addition of important nucleophiles, such as phenols and various $N$-hetereocycles. Assisted by the photoredox cycle, 1 or $3 \mathrm{~mol} \%$ Co catalyst was sufficient to attain a good yield in most cases. Mechanistically, quenching studies and stoichiometric reactions provided evidence for the involvement of photochemical oxidation of organocobalt(III) species. Based on these results along with the observed light-gated conversion, we anticipate possible spatial-temporal control on this bifurcated radical/polar reactivity, which may find applications in a broader context.

\section{ASSOCIATED CONTENT}

Supporting Information. Experimental procedures, characterization, crystal structure and spectra. This material is available free of charge via the Internet at http://pubs.acs.org.

\section{Corresponding Author}

*rongzhu@pku.edu.cn

\section{Notes}

The authors declare no competing financial interest.

\section{ACKNOWLEDGMENT}

Financial support was provided by the Natural Science Foundation of China (21901011), the "Thousand Talent Plan" Youth Program, and startup fund from College of Chemistry and Molecular Engineering, Peking University and BNLMS. The authors would like to thank Dr. Qing Zhang (PKU) for kind assistance, and Dr. Jie Su (PKU) for X-ray crystallography.

\section{REFERENCES}

(1) Markownikoff, W., I. Ueber die Abhängigkeit der verschiedenen Vertretbarkeit des Radicalwasserstoffs in den isomeren Buttersäuren. Annalen der Chemie und Pharmacie 1870, 153, 228.

(2) (a) Silvestri, R.; Artico, M.; La Regina, G.; Di Pasquali, A.; De Martino, G.; D'Auria, F. D.; Nencioni, L.; Palamara, A. T., Imidazole Analogues of Fluoxetine, a Novel Class of Anti-Candida Agents. J. Med. Chem. 2004, 47, 3924; (b) Cashman, J. R.; Voelker, T.; Johnson, R.; Janowsky, A., Stereoselective inhibition of serotonin re-uptake and phosphodiesterase by dual inhibitors as potential agents for depression. Bioorg. Med. Chem. 2009, 17, 337; (c) Gaul, M. et al. Indazole derivatives useful as glucagon receptor antagonists. US 2018/0065934 A1.

(3) (a) Rosenfeld, D. C.; Shekhar, S.; Takemiya, A.; Utsunomiya, M.; Hartwig, J. F., Hydroamination and Hydroalkoxylation Catalyzed by Triflic Acid. Parallels to Reactions Initiated with Metal Triflates. Org. Lett. 2006, 8, 4179; (b) Li, Z.; Zhang, J.; Brouwer, C.; Yang, C. G.; Reich, N. W.; He, C., Brønsted Acid Catalyzed Addition of Phenols, Carboxylic Acids, and Tosylamides to Simple Olefins. Org. Lett. 2006, 8, 4175.

(4) (a) Ananikov, V. P.; Beletskaya, I. P. Alkyne and Alkene Insertion into Metal-Heteroatom and Metal-Hydrogen Bonds: The Key Stages of Hydrofunctionalization Process. Top. Organomet. Chem. 2013, 43, 1-20; (b) Muller, T. E.; Hultzsch, K. C.; Yus, M.; Foubelo, F.; Tada, M., Hydroamination: Direct Addition of Amines to Alkenes and Alkynes. Chem. Rev. 2008, 108, 3795; For representative examples of $N$-heterocycle alkylation: (c) Sevov, C. S.; Zhou, J. S.; Hartwig, J. F., Iridium-Catalyzed, Intermolecular Hydroamination of Unactivated Alkenes with Indoles. J. Am. Chem. Soc. 2014, 136, 3200; (d) Ye, Y.; Kim, S. T.; Jeong, J.; Baik, M. H.; Buchwald, S. L., CuH-Catalyzed Enantioselective Alkylation of Indole Derivatives with Ligand-Controlled Regiodivergence. J. Am. Chem. Soc. 2019, 141, 3901.

(5) Crossley, S. W.; Obradors, C.; Martinez, R. M.; Shenvi, R. A., Mn-, Fe-, and Co-Catalyzed Radical Hydrofunctionalizations of Olefins. Chem. Rev. 2016, 116, 8912.

(6) For selected examples: (a) Isayama, S.; Mukaiyama, T., A New Method for Preparation of Alcohols from Olefins with Molecular Oxygen and Phenylsilane by the Use of Bis(acetylacetonato)cobalt(II). Chem. Lett. 1989, 18, 1071; (b) Kato, K.; Mukaiyama, T., Nitrosation of $\alpha, \beta$-Unsaturated Carboxamide with Nitric Oxide and Triethylsilane Catalyzed by Cobalt(II) Complex. Chem. Lett. 1990, 19, 1395; (c) Waser, J.; Carreira, E. M., Catalytic Hydrohydrazination of a Wide Range of Alkenes with a Simple Mn Complex. Angew. Chem. Int. Ed. 2004, 43, 4099; (d) Waser, J.; Nambu, H.; Carreira, E. M., Cobalt-Catalyzed Hydroazidation of Olefins: Convenient Access to Alkyl Azides. J. Am. Chem. Soc. 2005, 127, 8294; (e) Li, G.; Kuo, J. L.; Han, A.; Abuyuan, J. M.; Young, L. C.; Norton, J. R.; Palmer, J. H., Radical Isomerization and Cycloisomerization Initiated by $\mathrm{H} \bullet$ Transfer. J. Am. Chem. Soc. 2016, 138, 7698; (f) Crossley, S. W.; Barabe, F.; Shenvi, R. A., Simple, 
Chemoselective, Catalytic Olefin Isomerization. J. Am. Chem. Soc. 2014, 136, 16788; (g) Green, S. A.; Matos, J. L.; Yagi, A.; Shenvi, R. A., Branch-Selective Hydroarylation: Iodoarene-Olefin Cross-Coupling. $J$. Am. Chem. Soc. 2016, 138, 12779; (h) Matos, J. L. M.; Vasquez-Cespedes, S.; Gu, J.; Oguma, T.; Shenvi, R. A., Branch-Selective Addition of Unactivated Olefins into Imines and Aldehydes. J. Am. Chem. Soc. 2018, 140, 16976; (i) Ma, X.; Herzon, S. B., Intermolecular Hydropyridylation of Unactivated Alkenes. J. Am. Chem. Soc. 2016, 138, 8718; (j) Ma, X.; Herzon, S. B., Cobalt bis(acetylacetonate)-tert-butyl hydroperoxide-triethylsilane: a general reagent combination for the Markovnikov-selective hydrofunctionalization of alkenes by hydrogen atom transfer. Beilstein J. Org. Chem. 2018, 14, 2259; For Co-catalyzed hydroelementations via radical-polar crossover: (k) Shigehisa, H.; Aoki, T.; Yamaguchi, S.; Shimizu, N.; Hiroya, K., Hydroalkoxylation of Unactivated Olefins with Carbon Radicals and Carbocation Species as Key Intermediates. J. Am. Chem. Soc. 2013, 135, 10306; (1) Shigehisa, H.; Koseki, N.; Shimizu, N.; Fujisawa, M.; Niitsu, M.; Hiroya, K., Catalytic Hydroamination of Unactivated Olefins Using a Co Catalyst for Complex Molecule Synthesis. J. Am. Chem. Soc. 2014, 136, 13534; (m) Shigehisa, H.; Hayashi, M.; Ohkawa, H.; Suzuki, T.; Okayasu, H.; Mukai, M.; Yamazaki, A.; Kawai, R.; Kikuchi, H.; Satoh, Y.; Fukuyama, A.; Hiroya, K., Catalytic Synthesis of Saturated Oxygen Heterocycles by Hydrofunctionalization of Unactivated Olefins: Unprotected and Protected Strategies. J. Am. Chem. Soc. 2016, 138, 10597; (n) Date, S.; Hamasaki, K.; Sunagawa, K.; Koyama, H.; Sebe, C.; Hiroya, K.; Shigehisa, H., Catalytic Direct Cyclization of Alkenyl Thioester. ACS Catal. 2020, 10, 2039; (o) Shepard, S. M.; Diaconescu, P. L., Redox-Switchable Hydroelementation of a Cobalt Complex Supported by a Ferrocene-Based Ligand. Organometallics 2016, 35, 2446; (p) Vrubliauskas, D.; Vanderwal, C. D., Cobalt-Catalyzed Hydrogen Atom Transfer Induces Bicyclizations that Tolerate Electron-rich and -deficient Intermediate Alkenes. Angew. Chem. Int. Ed. 2020 doi: 10.1002/anie.202000252; For related stereoselective transformations: (q) Touney, E. E.; Foy, N. J.; Pronin, S. V., Catalytic Radical-Polar Crossover Reactions of Allylic Alcohols. J. Am. Chem. Soc. 2018, 140, 16982; (r) Discolo, C. A.; Touney, E. E.; Pronin, S. V., Catalytic Asymmetric Radical-Polar Crossover Hydroalkoxylation. J. Am. Chem. Soc. 2019, 141, 17527; (s) Ebisawa, K.; Izumi, K.; Ooka, Y.; Kato, H.; Kanazawa, S.; Komatsu, S.; Nishi, E.; Hiroya, K. and Shigehisa, H., Catalyst- and Silane- Controlled Enantioselective Hydrofunctionalization of Alkenes by TM-HAT and RPC Mechanism. ChemRxiv 2019, DOI: 10.26434/chemrxiv.9981395.v1; (t) Shen, X.; Chen, X.; Chen, J.; Sun, Y.; Cheng, Z.; Lu, Z., Ligand-promoted cobalt-catalyzed radical hydroamination of alkenes. Nature Communications 2020, 11, 783.; (u) Lu, S.; Niankai, F.; Brian G., E.; Wai-Hang, L.; Michael O., F.; Robert A., D. J.; Song, L., Dual Electrocatalysis Enables Enantioselective Hydrocyanation of Conjugated Alkenes. ChemRxiv 2019, DOI 10.26434/chemrxiv.9784625.v1.

(7) Zhou, X. -L.; Yang, F.; Sun, H. -L.; Yin, Y. -N.; Ye, W. -T.; Zhu, R., Cobalt-Catalyzed Intermolecular Hydrofunctionalization of Alkenes: Evidence for a Bimetallic Pathway. J. Am. Chem. Soc. 2019, 141, 7250.

(8) Yahata, K.; Kaneko, Y.; Akai, S., Cobalt-Catalyzed Intermolecular Markovnikov Hydroamination of Nonactivated Olefins: N2-Selective Alkylation of Benzotriazole. Org. Lett. 2020, 22, 598.

(9) (a) Vol'pin, M. E.; Levitin, I. Y.; Sigan, A. L.; Halpern, J.; Tom, G. M., Reactivity of organocobalt(IV) chelate complexes toward nucleophiles: diversity of mechanisms. Inorg. Chim. Acta 1980, 41, 271; (b) Zhu, R., Emerging Catalyst Control in Cobalt-Catalyzed Oxidative Hydrofunctionalization Reactions. Synlett 2019, 30, 2015.

(10) Vol'pin, M. E.; Levitin, I. Y.; Sigan, A. L.; Nikitaev, A. T., Current state of organocobalt(IV) and organorhodium(IV) chemistry. $J$. Organomet. Chem. 1985, 279, 263.

(11) For selected recent examples: (a) Liu, W. Q.; Lei, T.; Zhou, S.; Yang, X. L.; Li, J.; Chen, B.; Sivaguru, J.; Tung, C. H.; Wu, L. Z., Cobaloxime Catalysis: Selective Synthesis of Alkenylphosphine Oxides under Visible Light. J. Am. Chem. Soc. 2019, 141, 13941; (b) Chen, B.; Wu, L. Z.; Tung, C. H., Photocatalytic Activation of Less Reactive Bonds and Their Functionalization via Hydrogen-Evolution Cross-Couplings. Acc. Chem. Res. 2018, 51, 2512; (c) Yi, H.; Niu, L.; Song, C.; Li, Y.; Dou, B.; Singh, A. K.; Lei, A., Photocatalytic Dehydrogenative Cross-Coupling of Alkenes with Alcohols or Azoles without External Oxidant. Angew. Chem. Int. Ed. 2017, 56, 1120.

(12) Shevick, S. L.; Obradors, C.; Shenvi, R. A., Mechanistic Interrogation of $\mathrm{Co} / \mathrm{Ni}$-Dual Catalyzed Hydroarylation. J. Am. Chem.
Soc. 2018, 140, 12056

(13) (a) Chiang, L.; Allan, L. E.; Alcantara, J.; Wang, M. C.; Storr, T.; Shaver, M. P., Tuning ligand electronics and peripheral substitution on cobalt salen complexes: structure and polymerisation activity. Dalton Trans 2014, 43, 4295; (b) Kochem, A.; Kanso, H.; Baptiste, B.; Arora, H.; Philouze, C.; Jarjayes, O.; Vezin, H.; Luneau, D.; Orio, M.; Thomas, F., Ligand Contributions to the Electronic Structures of the Oxidized Cobalt(II) salen Complexes. Inorg. Chem. 2012, 51, 10557.

(14) Levitin, I.; Sigan, A. L.; Vol'pin, M. E., Electrochemical generation and reactivity of organo-cobalt(IV) and -rhodium(IV) chelates. J. Chem. Soc., Chem. Commun. 1975. 469. It was reported that an axial pyridine ligand had neglectable effect on the oxidation potential of an alkylcobalt(III) complex in MeCN.

(15) For details, please see SI-S29.

(16) Prier, C. K.; Rankic, D. A.; MacMillan, D. W. C., Visible Light Photoredox Catalysis with Transition Metal Complexes: Applications in Organic Synthesis. Chem. Rev. 2013, 113, 5322.

(17) Zhou, Q. Q.; Zou, Y. Q.; Lu, L. Q.; Xiao, W. J., Visible-Light-Induced Organic Photochemical Reactions through Energy-Transfer Pathways. Angew. Chem. Int. Ed. 2019, 58, 1586. Alterntively, this result could be attributable to the substantial absorption of Co complexes at $380 \mathrm{~nm}$.

(18) (a) Demarteau, J.; Debuigne, A.; Detrembleur, C., Organocobalt Complexes as Sources of Carbon-Centered Radicals for Organic and Polymer Chemistries. Chem. Rev. 2019, 119, 6906; (b) Liao, C.-M.; Hsu, C.-C.; Wang, F.-S.; Wayland, B. B.; Peng, C.-H., Living radical polymerization of vinyl acetate and methyl acrylate mediated by Co(Salen*) complexes. Polym. Chem. 2013, 4, 3098; (c) Liu, X.; Tian, L.; Wu, Z.; Zhao, X.; Wang, Z.; Yu, D.; Fu, X., Visible-light-induced synthesis of polymers with versatile end groups mediated by organocobalt complexes. Polym. Chem. 2017, 8, 6033.

(19) For an elegant example of on/off switch by chemical reduction/oxidation, see ref. 5o.

(20) Hansch, C.; Leo, A.; Taft, R. W., A survey of Hammett substituent constants and resonance and field parameters. Chem. Rev. 1991, 91, 165.

(21) Shigehisa, H., Functional Group Tolerant Markovnikov-Selective Hydrofunctionalization of Unactivated Olefins Using a Cobalt Complex as Catalyst. Synlett 2015, 26, 2479.

(22) Schmidt, A.; Beutler, A.; Snovydovych, B., Recent Advances in the Chemistry of Indazoles. Eur. J. Org. Chem. 2008, 2008, 4073.

(23) (a) Liang, Y.; Zhang, X.; MacMillan, D. W. C., Decarboxylative $\operatorname{sp}(3) \mathrm{C}-\mathrm{N}$ coupling via dual copper and photoredox catalysis. Nature 2018, 559, 83; (b) Song, F.; Xu, G.; Gaul, M. D.; Zhao, B.; Lu, T.; Zhang, R.; DesJarlais, R. L.; DiLoreto, K.; Huebert, N.; Shook, B.; Rentzeperis, D.; Santulli, R.; Eckardt, A.; Demarest, K., Design, synthesis and structure activity relationships of indazole and indole derivatives as potent glucagon receptor antagonists. Bioorg. Med. Chem. Lett. 2019, 29, 1974. 


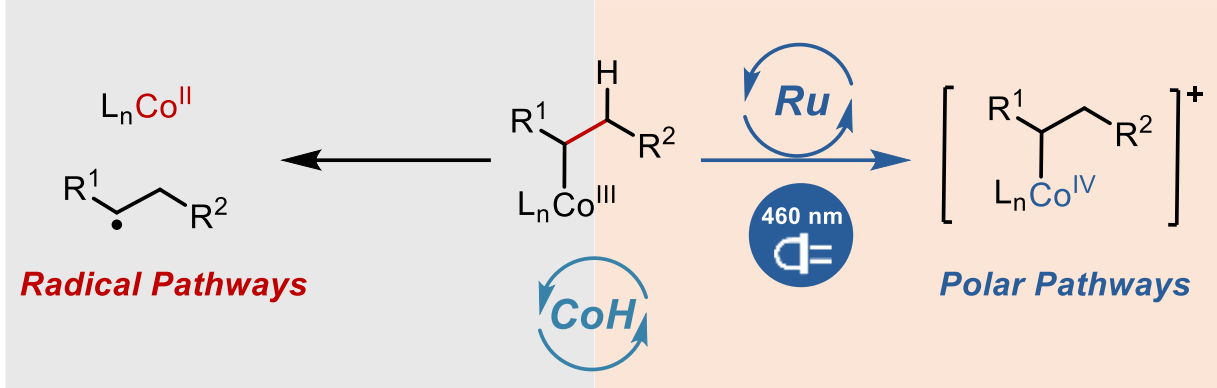

Light-Gated Reactivity

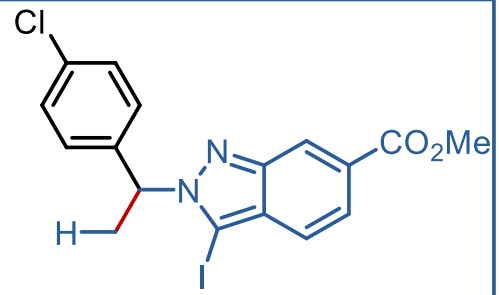

$3 \%$ [Co], 1\% [Ru], 69\% Yield Broad Scope Intermolecular Oxidative Hydrofunctionalization 